\title{
3D Imaging and Symmetry of the Calcaneal Bone
}

\section{Dominique André Misselyn ${ }^{1 *}$, Stijn De Buck ${ }^{2}$, Giovanni Matricali ${ }^{3}$ and Stefaan Nijs ${ }^{1}$}

${ }^{1}$ Department of Trauma Surgery, University Hospital, Leuven, Belgium

${ }^{2}$ Medical Imaging Research Center, University Hospital, Leuven, Belgium

${ }^{3}$ Department of Orthopedic Surgery, University Hospital, Leuven, Belgium

${ }^{*}$ Corresponding author: Dominique André Misselyn, Department of Trauma Surgery, University Hospital, Leuven, Belgium, Tel: +32495739784; Email: dominique.misselyn@uzleuven.be

Received date: September 19, 2018; Accepted date: October 08, 2018; Published date: October 18, 2018

Citation: Misselyn DA, Buck SD, Matricali G, Nijs S (2018) 3D Imaging and Symmetry of the Calcaneal Bone. J Clin Exp Orthop Vol 4. No 2:60.

Copyright: (C2018 Misselyn DA, et al. This is an open-access article distributed under the terms of the Creative Commons Attribution License, which permits unrestricted use, distribution, and reproduction in any medium, provided the original author and source are credited.

\section{Abstract}

Calcaneus fractures are painful and difficult to treat injuries. The quality of a good functional outcome is related to a good anatomical reconstruction. The contra-lateral uninjured bone is often used a reference to assess the severity of the damage and the quality of the repair. This is currently done with Böhler's angle which has to be drawn on X-Ray images. The authors of this study propose the use of $3 D$ reconstruction images of the hind foot and analyze the symmetry of healthy hind feet. Several measurements were recorded among the 3D reconstructions images of 46 pairs of uninjured feet: mean distances between facets and area of the calcaneal facet of the posterior subtalar joint, orientation angle of the posterior subtalar joint facet and talo-calcaneal angle in the space. The difference among all measurements was not statistically significant between the left and the right side, with a high power, which suggests a good symmetry of the calcaneus. Hence, 3D image of the contralateral uninjured calcaneus may be a reliable reference when treating calcaneal fractures.

Keywords: Calcaneus; 3D imaging; Principal component analysis

\section{Introduction}

Calcaneal fractures are very complex injuries, probably because of the intricate anatomy of this tarsal bone. Most fractures are intra-articular and when conservatively treated, are at risk of arthritis, probably because of the altered anatomy of the joint. Open reduction and internal fixation is currently the gold standard treatment of this complex injury [1]. Most authors agree that restoring the anatomy, especially the height and subtalar joint congruency is needed to achieve the best outcome. In case of intra-articular fracture, the major concern of the trauma surgeon is to repair the joint facet, giving back his anatomical position, with others words, avoiding step-off and giving a good orientation of this facet [2]. But this surgery remains challenging, probably because it is difficult to assess the quality of the reduction on classical X-Ray and 2D CT imaging. 3D imaging is a new imaging tool giving the opportunity to assess the severity of the fracture and prepare the surgical treatment $[3,4]$.

Based on the assumption of symmetry, it would make sense to use the contralateral uninjured calcaneus as a reference when treating a calcaneal fracture. The aim of this study is to confirm the symmetry of the calcaneal bones 3D images.

Several angles have been proposed to describe and quantify the general anatomy of the calcaneus, after trauma and after surgery: these angles are measured on X-Ray images, before and after treatment of the fractured calcaneus. Böhler's angle (BA) is the most used and was firstly described in 1931. Several others angles have been added: the critical angle of Gissane, the calcaneal inclination angle, the tibio-calcaneal angle, the talocalcaneal angle. A lower value of the BA supports the need of open reduction and internal fixation, a normalized value of the BA after surgery suggests a good anatomical repair, giving the hope of a better outcome.

Computerized 2D tomography was a major improvement in the radiological arsenal, however, this technology is not suitable for drawing angles: the landmarks of the BA are not anatomical.

In a study of 3D images of 12 pairs of healthy feet, Stephan described an algorithm to assess the symmetry of the calcaneus. His DICOM (digital imaging and communications in medicine) images were converted into 3D format .stl (stereo-lithography) with Amira ${ }^{\circledR}$ software. His computational tool compares 3D images of the calcaneus with several measurements. Measurements of the areas of the joint facets and of the joint facet orientation showed small intra-individual differences and large inter-individual variability. Because of the former, he concluded that the uninjured contralateral calcaneus 3D image may serve as anatomical reference in case of unilateral calcaneal fracture.

Stephan used the main axis of the calcaneus and of the talus as a references for his measurements of the orientation of the posterior joint facets. These axes were calculated by Principal Component Analysis (PCA). Measuring the orientation of the posterior subtalar joint facet with theses axes as reference 
makes sense: a good anatomical repair of the joint supposes a good orientation of his facet.

Exploiting the idea of Stephan to use the main axes of hind foot bones, the authors of this study propose the use of the angle between these axes, the "3D talo-calcaneal angle" or "3D TCA". It is measured by computing the angle between the main axis of the talus and the main axis of the calcaneus (Figure 1). Both axes are calculated by performing a principal component analysis (PCA) on the points describing the 3D surfaces of both the talus and calcaneus and selecting the axis along which the surface points have the largest distribution (i.e. with highest principal value), as described by Pearson [5]. Both 3D surfaces are constructed by manual segmentation of 3D medical image data obtained via CT imaging of the foot. Segmentation and 3D images are made with Mimics ${ }^{\circledR} 20$ software (Materialise ${ }^{\circledR}$, Leuven, Belgium).

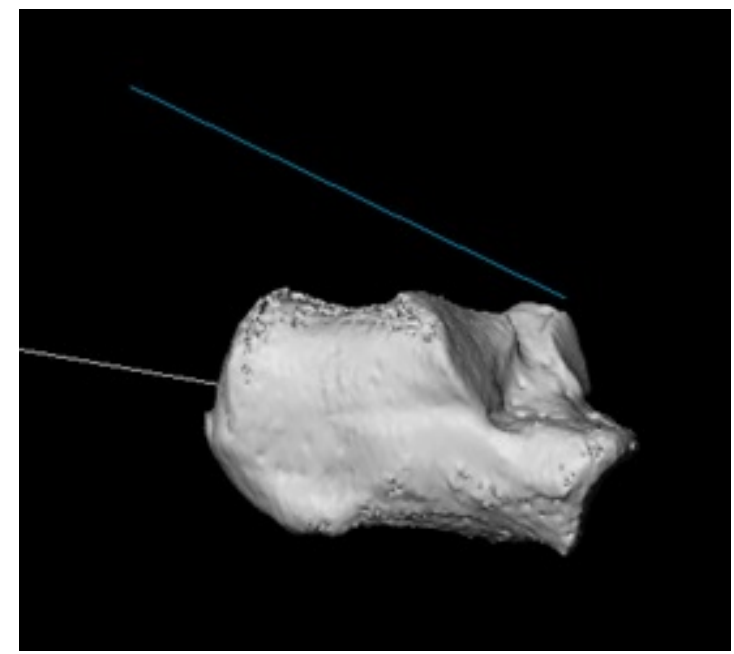

Figure 1 Example of main axis of the calcaneus in white. In blue the main axis of the talus (made invisible to allow a better view of the calcaneus).

\section{Materials and Methods}

DICOM images of bilateral lower limb of 46 patients (24 men, 22 women), were recorded over one year at the Radiology Department of the Leuven University Hospital. CT scans were performed for various reasons (preoperative workout of vascular or plastic surgery, virtual autopsies). Demographics were: 24 men, 22 women, mean age 54 years, range 17-85. All images were anonymized. Fracture of the hind foot was an exclusion criterion.

The CT images were recorded using the Aquilion ${ }^{\circledR}$ One Volume CT scanner (Toshiba ${ }^{\circledR}$, Tokyo, Japan), with a table pitch of 0.641 , gantry tilt of $0^{\circ}$, collimation of $0.5 \times 64$, current 100 $\mathrm{mA}$, voltage $135 \mathrm{kV}$, slice thickness of $0.5 \mathrm{~mm}$, slice increment of $0.5 \mathrm{~mm}$, and rotation time of 0.5 second. The radiation dose of CAT scan of extremities is far lower than in case of CAT scan of abdomen or chest and is considered as nearly without any deterministic and probabilistic risk.
The CT data were in DICOM format and 3D reconstruction of the hind foot done by choosing 226 Hounsfield Unit as threshold to reveal the bony structures of the calcaneus and talus with Mimics ${ }^{\circledR} 20$ (Materialise ${ }^{\circledR}$, Leuven, Belgium). The Region Growing function was used to obtain the masks of both tarsal bones. Every bone content was made uniform with the Multiple Slice Edit function, Live Wire. After manual segmentation, 3D surface meshes were made with the smoothing algorithm of Mimics ${ }^{\circledR}$. The 3D images of the left and right calcaneus and talus were imported into home-made software programmed in MeVisLab $^{\circledR}$ (MeVis Solution, Germany).

To compare left and right, the right calcaneus and talus were mirrored.

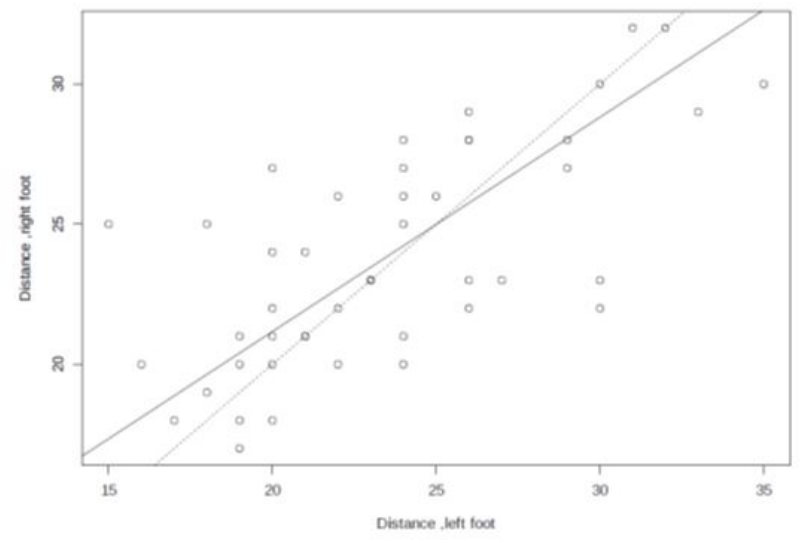

Figure 2 Graph of the average value of the distance between talar and calcaneal joint surface, left vs right. Orthogonal regression model shows a $45^{\circ}$ dashed line made by the equal value left vs right. The continuous line is the trend among the other points. The $p$-value shows there is no statistically significant difference between both lines.

$\mathrm{p}$-value of the difference between trend line and $45^{\circ}$ line: 0.0627 (Figure 2).

Several measurements were performed after manually marking the posterior subtalar joint (PSJ) facet on the 3D images. Based on this identification, the MeVisLab ${ }^{\circledR}$ software was able to measure the distance between every point of the marked joint facet of the calcaneus and its talar counterpart. This distance was calculated by measuring for every point of the joint facet the shortest distance to the talus joint. The average value of all the distances was calculated.

Another measurement is the whole surface of the PSJ facet.

The software also calculates the main axes of calcaneus and talus by PCA and measures the angle between both axes, the 3D talo-calcaneal angle, 3D-TCA. Orientation angle of the PSJ facet was also calculated. This last angle is the average angle between every normal vector at every point of the PSJ facet and the calcaneus main axis. All these calculations were thus automated and did not need any observer.

An observer was needed to manually outline the bony structures of the calcaneus and of the talus on DICOM images 
and to mark the PSJ on 3D images; this supposes a knowledge of the radiologic anatomy of the hind foot.

To assess the symmetry of the measurements in a pair of feet, we used the orthogonal regression model, because of the equal error variances, making the $\delta$ of the Deming regression (error-invariables model) equal to 1 , and the matched pairs t-test.

\section{Results}

All measurements of each calcaneus were compared with the contralateral side, left versus right: the area of the posterior subtalar joint facet ("area"), the mean distance between both facets of the posterior subtalar joint ("distance"), the orientation angle of the PSJ facet and the talo-calcaneal angle (3D-TCA).

Values were also statistically compared using orthogonal regression. The orthogonal regression model is an errors-invariables model which was chosen because of the equal uncertainty of the measures left and right.

Two lines were drawn on a graph with left and right values. One dashed line for subjects with equal values left and right, and one continuous line giving the trend calculated among the other points of the graph. P-value of the difference between both lines was calculated and was above the statistically significant value of 0.5 (Figure 3).
No statistically significant difference was found for all the measurements, which suggests there was symmetry between left and right calcaneus in our series of 46 pairs of feet.

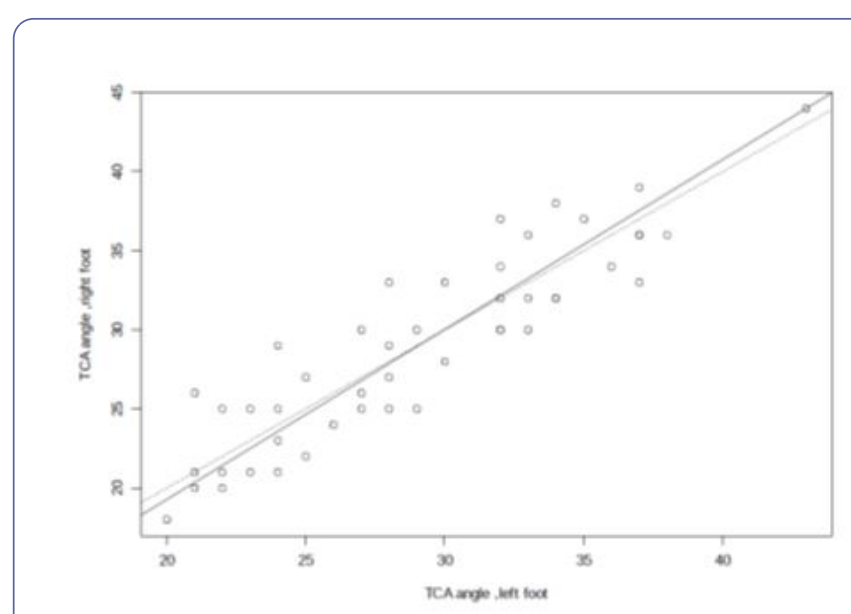

Figure 3 Graph of the 3D TCA angle, left vs right.

$\mathrm{p}$-value of the difference between the trend line and the $45^{\circ}$ line $=0.1259(\mathrm{M}), 0.1196(\mathrm{~F})$ mentioned in figures $4 \mathrm{a}$ and $\mathbf{4 b}$.

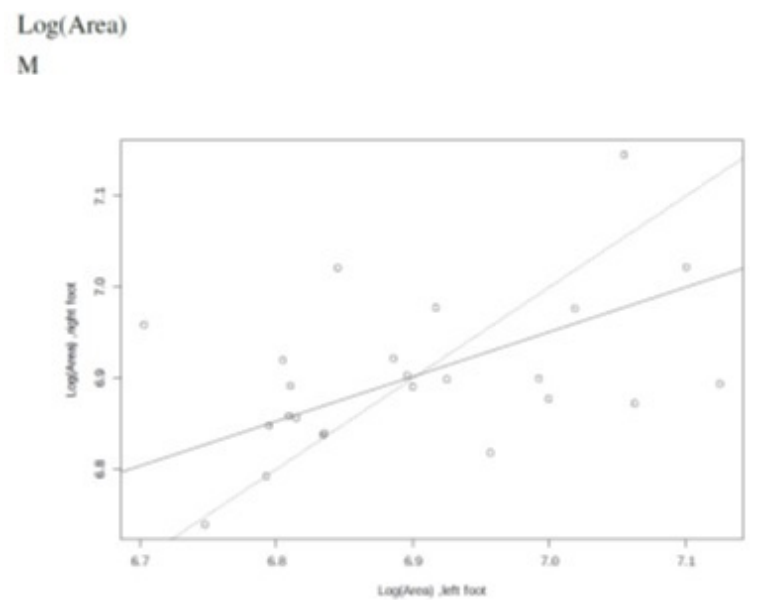

(a)

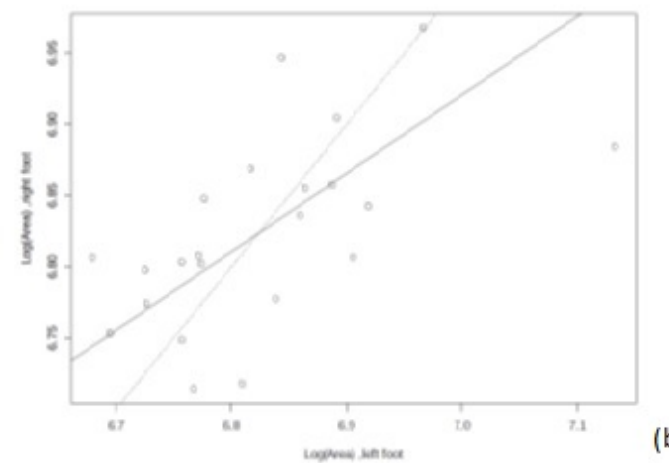

Figures $\mathbf{4 a}$ and $\mathbf{4 b}$ The dotted line is the $45^{\circ}$ line, with equal values. The continuous line is the orthogonal regression trend line (calculated by the statistical program). The first p-value is statistically significant, which means there is a relation between left and right values. The $p$-value of every orthogonal regression is not statistically significant, which means there is no clear difference between the two lines in all graphs.

Power analysis made by $\mathrm{G}^{*}$ Power software gives a power (1$\beta$ ) value of .80 for 1 degree of difference and even .97 for 1.5 degrees of difference (Figure 5).

\section{Discussion}

3D images of the calcaneus and talus provide striking views of these tarsal bones. Because of the high accuracy of these images and the supposed good symmetry of the human skeleton, many authors already use the contralateral side as reference in clinical practice, such as for pre-shaping an osteosynthesis plate [6].
Because of the complexity of the anatomy, the difficulty of the procedure and the subcutaneous position of the implant, it makes sense to pre-shape the osteosynthesis plate on the mirrored contralateral calcaneus. The plate is not only a fixation but also a preoperative and intraoperative guide. However, the symmetry of calcaneal 3D images has not been established on such ample evidence.

3D imaging is gaining popularity and Qiang was able to draw BA on 3D images of normal calcaneus with good reproducibility [7]. In place of the BA, we proposed to use the 3D-TCA and 3D PSJ orientation angle. These angles, if it may be of clinical use, 
needs to be symmetrical and have a normal and reproducible value.

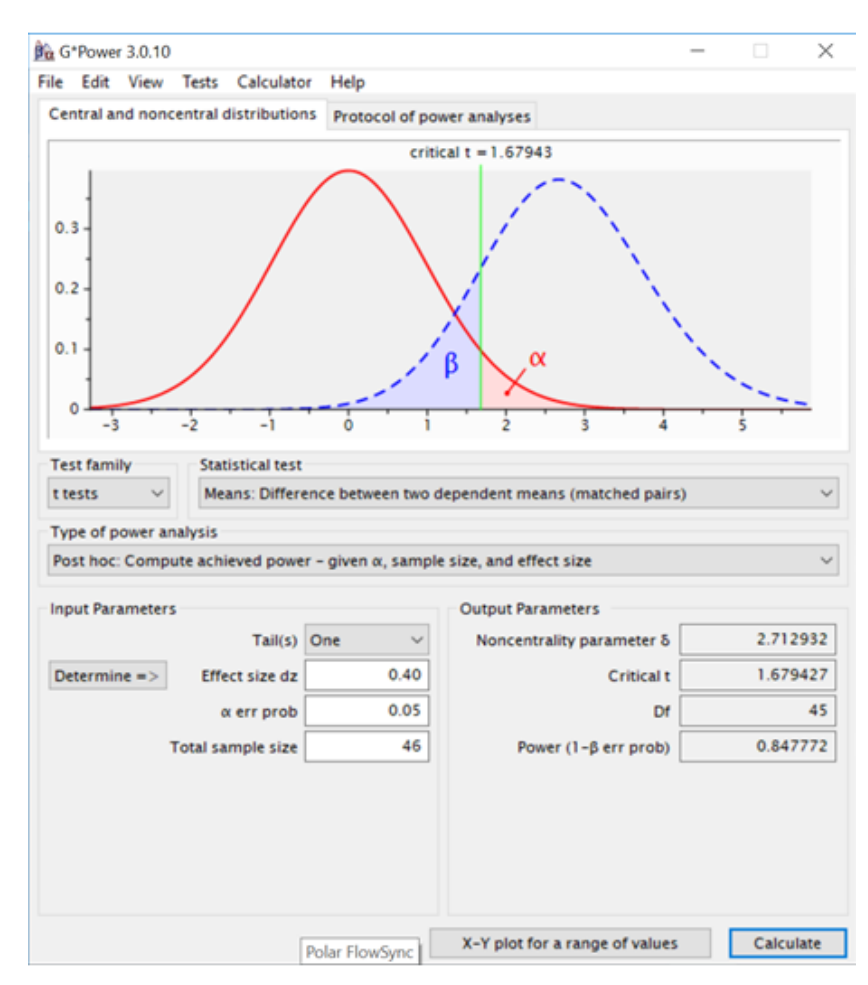

Figure 5 Symmetry of the TCA angles was also assessed with a matched pairs t-test. As we consider the null-hypothesis of a difference between left and right being 0 , we find .91 as $p$ value, which allows us to accept it.

Given a good segmentation of calcaneus and talus, the advantage of these measures is that it can be computed in an semi-automated way. Since it is based on all the points describing the calcaneus/talus, which often amounts to several tens of thousands, the measure is likely less prone to user input variability.

Errors may still occur because of the manual operations involved in the creation of the 3D surface of both bony structures which assumes a certain level of knowledge of radiological anatomy, and experience in using the segmentation software.

It makes sense to use a 3D TCA: the orientation of the calcaneus and of the talus are a major concern for the surgeon during hind foot surgery. It make also sense to use 3D orientation angle of the PSJ facet: anatomical repair of the PSJ facet is the main concern of the surgeon and this facet is a wellknown anatomical structure, easy to identify on 3D images with his clear borders. After conservative treatment of a calcaneus fracture, this bone is often too broad and patient complaints are often related to misalignment of the hind-foot. The use of the main axes on $3 D$ images is probably better than the use of a lateral X-Ray view.

The use of only one main axis is better than a system with 3 axes: the two additional ones may switch because of the relative symmetry of the bone itself, and the use of the positioning of the foot as reference may not be always possible when the foot is very painful, like after trauma or after surgery. The main axis of every bone is also easier to determine and calculate, and is already in use for other bones [8-10].

Table 1: Mean values of distance, areas, orientation angle of the PSJ and 3D TCA of left and right hind feet.

\begin{tabular}{|l|l|l|}
\hline Measurements & Left(SD) & Right(SD) \\
\hline Sabtalar joint distances(mm) & $2.4(0.47)$ & $2.4(0.39)$ \\
\hline Areas subtalar joint( $\left.\mathrm{mm}^{2}\right)$ & $964(114)$ & $961(84)$ \\
\hline Orientation angle PSJ(degrees) & $100(6.6)$ & $101(6.3)$ \\
\hline 3D talo cancanal angle(degrees) & $29(5.69)$ & $29(5.97)$ \\
\hline
\end{tabular}

Intuitively, we may determine that calcaneus are symmetrical, but when considering 3D images, there are not many studies giving evidence of this symmetry, probably because of the high complexity in the internal structures of the $3 D$ images. When using axis calculated by PCA, one must deal with uniformly filled structures, because a small defect can heavily weigh on the average direction of the axis.

Unlike Stephan, we considered only the PSJ facet, because it is by far the biggest of the four joint facets and it is the most important one for the surgeon.

In the current study, we performed several measurements on 3D images of calcaneal bone pairs and none of them showed statistically significant differences between left and right calcaneus (Table 1). This is an objective argument in favor of symmetry.

Orthogonal regression model was the best statistical model in regression analysis of symmetry because of the standard error of measurement at both sides. One may object that it cannot rule out a difference in dimensions, such as one bone having the same shape with height, length and width 2 times smaller, but this occurrence is intuitively unrealistic.

Orthogonal regression statistical test proves the symmetry of the TCA and orientation angles. With the t-test of paired series, we found a $p$ value that allows acceptance of the null hypothesis of no statistically significant difference between angles of both sides. Accepting a null hypothesis may be criticized, but the power analysis confirms it with a very high probability.

Several authors already proposed the use of principal axis $[9,11]$ of isolated bone as a reference when working on 3D images. Unlike Stephan, our main axes calculation were based on surface and not on volume. Most authors agree that the difference between both is minimal.

The use of the 3D talo-calcaneal angle and of the PSJ orientation angle has potential added value: they are certainly affected by a fracture because of the severe change in the anatomy. They are also probably better than the average distance of the area of the joint facet whose average value may be influenced by small persisting gaps in the joint surface after osteosynthesis. Their measurement is computed, which renders it more reproducible; the measurement of the PSJ orientation 
angle is calculated after marking the posterior joint facet on 3D image: this facet is a well-known anatomical structure, easy to identify and to outline. The orientation angle may be able to quantify the quality of the reduction of the PSJ facet. The uninjured contralateral side may be used as reference when mirrored. This means more radiation, but scanning both feet means a very low radiation dosis.

The limiting factor is the identification of the calcaneus and tarsus on DICOM images, which still relies on knowledge of anatomy, especially radiological anatomy. In the future, we may assume that the $3 \mathrm{D}$ reconstruction process will become more and more automated, which will limit observer bias.

\section{Conclusion}

The calcaneus has a complex anatomy and segmented 3D image gives a very realistic overview of this tarsal bone. Several measurements on 3D images of 46 pairs healthy feet showed a good symmetry between left and right. It supports the use of the uninjured side as a reference when treating fractured calcaneus. The authors describe angles, which can be used in evaluating the repair of the anatomy after osteosynthesis, like Böhler's angle on X-Ray images. To our knowledge, this is the first study examining symmetry of 3D images of calcaneal bones with so many subjects. If confirmed on patients who underwent osteosynthesis of DIACF, it may become a new indicator in the treatment of this difficult to treat injury.

\section{References}

1. Buckley R, Tough S, Mc Cormack R, Pate G, Leighton R, et al. (2002) Operative compared with non-operative treatment of displaced intra-irticular calcaneal fractures: a prospective, randomized, controlled multicenter trial. J Bone Joint Surg Am 84: 1733-1744.
2. Kurozumi T, Jinno $Y$, Sato T, Inoue H, Aitani T, et al. (2003) Open reduction for intra-irticular alcaneal fractures: evalution using computed tomography. Foot Ankle Int 24: 942-948.

3. Schepers T, Misselyn D (2017) 3D printing calcaneal fractures: continuously improving our care by making a complex problem tangible. J Invest Surg 9: 1-2.

4. Zheng W, Tao Z, Lou Y, Feng Z, Li H, et al. (2017) Comparison of the conventional surgery and the surgery assisted by $3 \mathrm{D}$ printing technology in the treatment of calcaneal fractures. J Invest Surg 19: 1-11.

5. Pearson K (1901) On lines and planes of closest fit to systems of points in space. Philosophical Magazine 2: 559-572.

6. Chung KJ, Hong DY, Kim YT, Yang I, Park YW et al. (2014) Preshaping plates for minimally invasive fixation of calcaneal fractures using a real-size 3D-printed model as a preoperative and intra-operative tool. Foot Ankle Int 35:1231-1236.

7. Qiang M, Chen Y, Zhang K, Li H, Dai H (2014) Measurement of three-dimensional morphological characteristics of the calcaneus using CT image post-processing. J Foot Ankle Res 7: 19.

8. Beimers L, Tuijthof GJ, Blankevoort L, Jonges $R$, Maas $M$, et al. (2008) In-vivo range of motion of the subtalar joint using computed tomography. J Biomech 4: 1390-1397.

9. Stephan D, Panzer S, Göttlinger M, Augat P (2013) Analysis of the intra-individual differences of the joint surfaces of the calcaneus. Comput Methods Biomech Biomed Engin 17: 1635-1641.

10. Volk I, Gal, Peleg E, Almog G, Luria S (2017) Three-dimensional comparison of alternative screw positions versus actual fixation of scaphoid fractures. Injury 48: 1183-1189.

11. Parr W, Chatterjee H, Soligo C (2012) Calculating the axes of rotation for the subtalar and talocrural joints using 3D bone reconstructions. J Biomech 45: 1103-1107. 